Early Draft (please do not cite without permission)

\title{
Effective Carbon Prices and Sub-Global Climate Cooperation
}

\author{
Goran Dominioni
}

\begin{abstract}
Scholarly and policy interest in carbon pricing coalitions is growing. Existing research analyzes design features that can increase the environmental effectiveness and political resilience of coalitions centered around carbon taxes and carbon markets (i.e. explicit carbon pricing). This article is the first that analyzes the advantages and disadvantages of building carbon pricing coalitions around effective carbon pricing compared to the standard design that focuses on explicit carbon pricing. In this article, measures of effective carbon prices include carbon prices implemented via carbon taxes, carbon markets, fuel taxes, and fossil fuel subsidies reforms. The article describes four design options to build carbon pricing coalitions - three built on measures of effective carbon pricing and one that focuses exclusively on explicit carbon pricing-and benchmarks them against six criteria. The key results are that building carbon pricing coalitions around effective carbon prices has various advantages over the most common alternative discussed in the literature. These advantages include higher transparency, broader participation, higher legitimacy of the coalition, and more substantial involvement of Finance Ministries in climate change mitigation. These advantages might translate incomparable or even higher environmental effectiveness than coalitions that focuses exclusively on explicit carbon pricing.
\end{abstract}

